\title{
„Arbeitsscheue Volksgenossen“1 Leistungsbereitschaft als Kriterium der Inklusion und Exklusion
}

Am 30. September 1933 ordnete der Landrat von Jüterbog-Luckenwalde gegen den Arbeiter Hermann S. „Schutzhaft“ an. Die Haftbegründung lautete:

Sie haben wiederholt die Ihnen zugewiesenen Arbeitsgelegenheiten verweigert [beziehungsweise] Ihre Arbeitsstätte nach wenigen Tagen wieder verlassen und damit zu erkennen gegeben, dass Sie nicht arbeiten, sondern auf Kosten der Allgemeinheit leben wollen. [...] Durch Ihr Verhalten widersetzten Sie sich der dringlichsten Aufgabe der nationalen Regierung, die Arbeitslosigkeit zu bekämpfen, und kennzeichnen sich dadurch als volksund staatsfeindliches Element. Ihre Inschutzhaftnahme ist daher gerechtfertigt und erforderlich. $^{2}$

Hermann S. wurde in das KZ Oranienburg eingewiesen. Die Dauer seiner Haft ist unbekannt.

Die „Schutzhaftanordnung“ des Landrates von Jüterbog-Luckenwalde belegt, dass bereits im ersten Jahr der NS-Herrschaft vermeintliche oder tatsächliche Arbeitsunwilligkeit als „volks“- beziehungsweise „staatsgefährdend“ gewertet und verfolgt wurde. Sie macht darüber hinaus deutlich, dass bereits zu dieser Zeit „arbeitsscheues“ Verhalten den Anlass für eine KZ-Einweisung bildeten konnte. Und sie verweist auf eine „Schutzhaftpraxis“, die die Zweckbestimmung der „Reichstagsbrandverordnung“, nämlich der „Abwehr kommunistischer staatsgefährdender Gewaltakte“3 $\mathrm{zu}$ dienen, soweit ausdehnte, dass darunter auch Verhaltensweisen subsummiert werden konnten, die ihrem Wesen nach zwar unpolitisch waren, aber sozial Anstoß erregten.

„Schutzhaftfälle“, in denen beispielsweise eine nicht-sesshafte Lebensweise, „respektloses“ Gebaren gegenüber Amtspersonen, Alkoholismus oder das

1 Ulrich Sondermann-Becker, „Arbeitsscheue Volksgenossen“. Evangelische Wandererfürsorge in Westfalen im „Dritten Reich“. Eine Fallstudie, Bielefeld 1995. Die Kombination des Adjektivs „arbeitsscheu“ mit dem Substantiv „Volksgenossen“ kommt in den Quellen nicht vor und ist innerhalb des Gedankengebäudes der NS-Ideologie auch begrifflich undenkbar. Der Buchtitel von Ulrich Sondermann-Becker wurde hier aufgegriffen, weil gerade die Verbindung dieser beiden einander ausschließenden Termini auf das paradoxe Spannungsverhältnis verweist, das der rassentheoretischen Annahme von der erbbiologischen „Minderwertigkeit“ bestimmter Individuen und Gruppen innerhalb der ,arischen Rasse“ inhärent ist.

2 Brandenburgisches Landeshauptarchiv Potsdam, Rep. 35 G, „Schutzhaftbefehl“, 30.9.1933.

3 Verordnung des Reichspräsidenten zum Schutz von Volk und Staat vom 28.2.1933, in: RGBl. I, S. 83. 
Bestreiten des Lebensunterhaltes durch Bettelei, mobiles Kleingewerbe oder Sexarbeit den Grund für die Inhaftierung bildeten, sind schon für das Jahr 1933 aus allen Gebieten des Reiches überliefert. Sie erreichten zwar längst nicht den Umfang wie die Verhaftung von Regime-Gegnern. Dennoch stellte die KZ-Einweisung von Hermann S. im September 1933 keinen Einzelfall dar - zumal keine zwei Wochen zuvor die „Bettlerrazzia“ stattgefunden hatte, bei der reichsweit über 10000 Personen polizeilich kontrolliert worden waren. Einige von ihnen waren den Amtsgerichten vorgeführt und zu kurzen Haftstrafen verurteilt worden. Andere hatte man ohne gerichtliches Strafverfahren in die frühen KZ gesperrt. ${ }^{4}$ Im Anschluss an die „Bettlerrazzia“, um die Jahreswende 1933/34, war dann eine Ausweitung der „Schutzhaftanordnungen“ im Falle sozialer Devianz zu beobachten. Allerdings schoben die „Schutzhafterlasse“ dieser Entwicklung im April 1934 einen Riegel vor. Wie im Folgenden noch zu zeigen sein wird, griffen die Behörden bei der KZ-Einweisung sozialer Randgruppen aber schon bald auf andere Mittel zurück, beispielsweise auf den $\S 20$ der „Reichsverordnung über die Fürsorgepflicht“5 von 1924 oder die polizeiliche „Vorbeugungshaft“.

Obgleich das Vorgehen gegen normabweichendes Sozialverhalten in der ersten Hälfte der 1930er-Jahre nicht zentral gesteuert war, korrespondierte es doch mit grundlegenden Elementen der NS-Ideologie: Die nationalsozialistische „Volksgemeinschaft“ war nicht nur als „rassisch“, sondern auch als sozial homogene Einheit und „völkische Leistungsgemeinschaft“6 konzipiert. Leistungsbereitschaft beziehungsweise deren Gegenteil, die „Arbeitsscheue“, dienten als Unterscheidungsmerkmal, um zwischen den „wertvollen“ und den „minderwertigen Volksgenossen“ zu differenzieren. Leistungsbereitschaft war daher ein zentrales Kriterium der Inklusion beziehungsweise Exklusion.

Auf der einen Seite sollte der Leistungswille der „Volksgenossen“ durch vielfältige kollektive und individuelle Anreize geweckt und prämiert werden. ${ }^{7}$ $\mathrm{Zu}$ denken ist hier an das Freizeit- und Ferienprogramm „Kraft durch Freude“, an die Betriebswettkämpfe um den Titel „nationalsozialistischer Musterbe-

4 Wolfgang Ayaß, „Asoziale“ im Nationalsozialismus, Stuttgart 1995, S. $19-47$.

5 Im behördlichen Sprachgebrauch benutzte man häufig die Abkürzung „Reichsfürsorgepflichtverordnung“ (RFV). Die RFV wurde im Verlauf der Weimarer Republik mehrfach abgeändert. Hier liegt die Fassung von 1932 zu Grunde: Peter A. Baath, Verordnung über die Fürsorgepflicht vom 13. Februar 1924 einschließlich der für Voraussetzung, Art und Maß der öffentlichen Fürsorge geltenden Reichsgrundsätze und der Nebengesetze sowie der einschlägigen landesrechtlichen Vorschriften, Berlin 1933.

6 Günter Morsch, Arbeit und Brot. Studien zu Lage, Stimmung, Einstellung und Verhalten der deutschen Arbeiterschaft 1933-1936/37, Frankfurt a.M. 1993, S. 33-38.

7 Frank Bajohr/Michael Wildt, Einleitung, in: dies. (Hrsg.), Volksgemeinschaft. Neue Forschungen zur Gesellschaft des Nationalsozialismus, Frankfurt a.M. 2009, S. 7-23. 
trieb“ oder, als geschlechtsspezifische Prämie, auch an das Mutterverdienstkreuz. Leistungsverweigerung, auf der anderen Seite, wurde unnachgiebig verfolgt und geahndet. Mit dem Einsetzen des Vernichtungsprogramms Anfang der 1940er-Jahre fielen dann auch jene Personen, gegen die man aufgrund ihres normabweichenden Sozialverhaltens KZ-Haft verhängt hatte, den systematischen Tötungen zum Opfer. ${ }^{8}$ Dennoch verwiesen die nach dem Machtantritt der Nationalsozialisten gegen „Asoziale“ und „Berufsverbrecher“ ergriffenen Maßnahmen zunächst auf Traditionen, die weit in die Zeit vor 1933 zurückreichten.

Der Fokus meiner weiteren Ausführungen wird auf den Mechanismen der Exklusion aus der nationalsozialistischen „Volks“- und „Leistungsgemeinschaft“ liegen beziehungsweise noch spezifischer: auf der Verhängung von KZ-Haft gegen die sogenannten „Asozialen“ und „Berufsverbrecher“. Konzentrieren werde ich mich dabei auf die Zeit zwischen dem Machtantritt der Nationalsozialisten und den Massenrazzien gegen „Asoziale“ und „Berufsverbrecher“ in den Jahren 1937/38. Mit Blick auf diese Eingrenzung muss allerdings zweierlei hervorgehoben werden: Erstens betrafen die Repressionen aufgrund mangelnder Leistungsbereitschaft nicht ausschließlich die Angehörigen der traditionellen gesellschaftlichen Randgruppen. ${ }^{9}$ Und zweitens war die KZ-Haft bei weitem nicht das einzige Sanktionsmittel der Verfolgungsbehörden. Hinzu kamen die rechtliche und wirtschaftliche Schlechterstellung der Betroffenen, beispielsweise durch die schrittweise Ausgrenzung aus der Wohlfahrtsunterstützung, die Einberufung zu Notstandsarbeiten, zum Reichsarbeitsdienst, ja sogar die Einweisung in Lager und Anstalten der geschlossenen Fürsorge. Nicht zuletzt drohte den Betroffenen auch die Zwangssterilisation oder -kastration.

\footnotetext{
8 Ab 1940 waren auch als „Asoziale“ klassifizierte Personen von den „Euthanasie“-Morden betroffen. Außerdem war ihr Leben, ebenso wie das der Mehrfachstraftäter, in den KZ durch die mörderischen Arbeitsbedingungen und die Selektionen bedroht.

9 Detlev Humann zeigt, dass sich die Verfolgungsmaßnahmen aufgrund von unterstellter oder tatsächlicher Leistungsverweigerung auch gegen Angehörige anderer Gesellschaftsschichten richten konnten, insbesondere dann, wenn sie in nationalsozialistische Großprojekten wie dem Arbeitsdienst oder der Landhilfe beschäftigt waren: Detlev Humann, Ordentliche Beschäftigungspolitik? Unterstützungssperren, Drohungen und weitere Zwangsmittel bei der „Arbeitsschlacht“ der Nationalsozialisten, in: VfZ (2012) 1, S. 33-67, hier S. 61-66.
} 


\section{1 „Arbeitsscheue“ als Zeichen erblicher „Minderwertigkeit“. Diskursive und praktische Anknüpfungspunkte aus der Zeit vor 1933}

Der Historiker Detlev Peukert unterschied einst zwischen einem „ethnischen“ und einem „eugenischen Rassismus“. ${ }^{10}$ Der Vorteil einer solchen Differenzierung liegt darin, dass sie zwar einerseits die soziale von der interkulturellen Stoßrichtung des Rassismus trennt, gleichzeitig aber deren inneren Zusammenhang kennzeichnet.

Während sich der ethnische Rassismus nach „außen“, gegen die als „artfremd“ und daher nicht zur nationalsozialistischen „Volksgemeinschaft“ gehörig erachteten Personen und Gruppen richtete, bezog sich der eugenische Rassismus auf die angeblich „minderwertigen“ Mitglieder der eigenen „arischen Rasse“. Für eine an rassistischen Prämissen ausgerichtete Politik, wie sie das NS-Regime verfolgte, eröffnete sich mit der eugenischen Stoßrichtung allerdings auch eine zentrale Problematik: Ließen sich die vermeintlich ,artfremden“ Individuen noch verhältnismäßig leicht anhand von intersubjektiv überprüfbaren Kriterien wie dem Herkunfts- beziehungsweise Geburtsland, dem Glaubensbekenntnis oder dem Stammbaum bestimmen, war die Frage der Identifizierung im Hinblick auf die „minderwertigen“, aber dennoch „arischen Volksgenossen“ ungleich schwieriger $\mathrm{zu}$ entscheiden. Bekanntlich verortete man die Ursache der „Minderwertigkeit“ im degenerierten „Erbgut“, dem „Blut“ oder in nicht näher lokalisierbaren, aber auf jeden Fall angeborenen „Rasseeigenschaften“ - in Eigenheiten also, die sich dadurch auszeichneten, dass sie äußerlich unsichtbar waren. Um dennoch die sozialpolitischen Implikationen, die man meinte aus der „erb“- und „rassenbiologisch“ fundierten Gesellschaftstheorie ableiten zu können, in praktisch-politische Programme umzusetzen, war man darauf angewiesen, die Träger „minderwertigen“ Erbgutes - und damit der als „schlecht“ oder „nachteilig“ erachteten sozialen Eigenschafen und Verhaltensweisen - mögliches einfach und zweifelsfrei zu identifizieren.

10 Detlev Peukert, Alltag und Barbarei. Zur Normalität des Dritten Reiches, in: Gewerkschaftliche Monatshefte (1987) 3, S. 142-153, hier S. 148; ders., Die Genesis der „Endlösung“ aus dem Geiste der Wissenschaft, in: Siegfried Blasche et al. (Hrsg.), Zerstörung des moralischen Selbstbewusstseins. Chance oder Gefährdung? Praktische Philosophie in Deutschland nach dem Nationalsozialismus, Bad Homburg 1988, S. 24-48, hier S. 25, 38; ders., Volksgenossen und Gemeinschaftsfremde. Anpassung, Ausmerze und Aufbegehren unter dem Nationalsozialismus, Köln 1982, S. 246-279. 
Es war unter anderem diese anwendungsbezogene Problematik, die zur Entwicklung einer nahezu unüberschaubaren Fülle von Ansätzen zur „rassenbiologischen“ Klassifikation der Bevölkerung führte. So war es schon im 19. Jahrhundert eines der Hauptanliegen der gerade im Entstehen begriffenen Kriminologie, anhand physischer und charakterlicher Merkmale sogenannte „Verbrechertypologien“ zu bilden, mit deren Hilfe man unter anderem hoffte, den „geborenen Verbrecher“ bestimmten zu können. ${ }^{11}$ Doch schlugen alle Ansätze, einen Katalog allgemeinverbindlicher Merkmale mit daran geknüpfter Kategorisierung festzuschreiben, letztlich fehl. Stattdessen kursierte eine Vielzahl konkurrierender Erklärungen für die Ursachen des Verbrechens und ebenso zahlreiche „Verbrechertypologien“.12 Dieses Scheitern kriminologischer und „erbbiologischer“ Forschung könnte erklären, warum man in der Praxis auf die Leistungsbereitschaft beziehungsweise „Arbeitsscheue“ als Bestimmungsmerkmal der „erb“- und „rassenbiologischen Minderwertigkeit“ zurückgriff. Im „erb“- und „rassenbiologisch“ inspirierten Denken galten als „arbeitsscheu“ aufgefasste Verhaltensweisen als Ausdruck der angeborenen „Minderwertigkeit“", ${ }^{13}$ die durch das degenerierte Erbgut verursacht wurde. Im Gegensatz zu diesem war das „arbeitsscheue“ Verhalten aber sichtbar.

Wie eingangs erwähnt knüpften die Exklusions- und Verfolgungspraktiken, die sich während des Nationalsozialismus gegen die als „minderwertig“ und „arbeitsscheu“ klassifizierten Personen und Gruppen richteten, an Diskurse und Praktiken an, die zeitlich noch viel weiter zurück lagen, als durch den Verweis auf die Kriminologie bereits angedeutet. Einige Verbindungslinien lassen sich bis in die Phase der Frühindustrialisierung zurückverfolgen, in der sich Konzepte und Einrichtungen der Sozialdisziplinierung herausbildeten, die wie die Arbeitshäuser bis weit ins 20. Jahrhundert hinein wirkungsmächtig sein sollten. ${ }^{14}$ Wie ich im Folgenden zeigen werden, lassen sich abgesehen von solch

11 Cesare Lombroso, Der Verbrecher in anthropologischer, ärztlicher und juristischer Beziehung, Hamburg 1887; ders., Der Verbrecher in anthropologischer, ärztlicher und juristischer Beziehung, Hamburg 1890.

12 Robert Heindl, Der Berufsverbrecher. Ein Beitrag zur Strafrechtsreform, Berlin 1926, S. 117169; Franz von Liszt, Der Zweckgedanke im Strafrecht [1882], in: ders., Aufsätze und kleinere Monographien, Hildesheim 1999, S. 126-179, hier S. 165-173; Silviana Galassi, Kriminologie im Deutschen Kaiserreich. Geschichte einer gebrochenen Verwissenschaftlichung, Stuttgart 2004, S. 351-360.

13 Hier überschneiden sich der ethnische und der eugenische Rassismus, denn auch den Zielgruppen des ethnischen Rassismus wurde häufig „Arbeitsscheue“ unterstellt. Erinnert sei beispielsweise an das Bild vom „faulen Juden“ oder an die Gegenüberstellung des „schaffenden“ und des „raffenden“ Kapitals, wobei man ersteres mit den „Ariern“ und letzteres mit den Juden identifizierte.

14 Wolfgang Ayaß, Die „korrektionelle Nachhaft“. Zur Geschichte der strafrechtlichen Arbeitshausunterbringung in Deutschland, in: ZNR 15 (1993), S. 184-201; Gerhard Pfeisinger, 
weitgreifenden Traditionslinien aber auch direkte konzeptionelle Vorläufer identifizieren, auf welche die Akteure nach dem Machtantritt der Nationalsozialisten ihr Vorgehen gegen soziale Außenseiter und Mehrfachstraftäter stützen konnten. Sie finden sich vor allem innerhalb der Strafrechtsreformbewegung ${ }^{15}$ und im Bereich der Wohlfahrtspflege, genauer gesagt: in der Praxis des fürsorgerechtlichen Arbeitszwangs und der Pflichtarbeit ${ }^{16}$ sowie in den Diskussionen über ein „Bewahrungsgesetz“. ${ }^{17}$

\section{Die erste Phase der nationalsozialistischen Verfolgung von „Asozialen“ und „Berufsverbrechern“ 1933 bis 1937. Institutionengefüge und Rechtsgrundlagen}

Bis zum „Grundlegenden Erlass über die vorbeugende Verbrechensbekämpfung durch die Polizei““ ${ }^{18}$ mit dem das Reichsinnenministerium am 14. Dezember 1937 eine einheitliche Regelung der polizeilichen „Vorbeugungshaft“ schuf, und den anschließenden Massenverhaftungen durch die Gestapo und die Kriminalpolizei 1938, waren die rechtlichen Bestimmungen zur KZ-Einweisung jener Gruppen, die im Jargon der Verfolgungsbehörden gemeinhin als „Asoziale“ und „Berufsverbrecher“ bezeichnet wurden, den Landesregierungen überlassen. Kennzeichnend für diese erste Verfolgungsphase waren daher die Heterogenität der Maßnahmen und die Vielfalt der in die Verfolgung involvierten Behörden. ${ }^{19}$

Arbeitsdisziplinierung und frühe Industrialisierung 1750-1820, Wien 2006; Hubert Treiber/Heinz Steinert, Die Fabrikation des zuverlässigen Menschen. Über die „Wahlverwandtschaft" von Kloster- und Fabrikdisziplin, Münster 2005.

15 Christian Müller, Verbrechensbekämpfung im Anstaltsstaat. Psychiatrie, Kriminologie und Strafrechtsreform in Deutschland 1871-1933, Göttingen 2004.

16 Ayaß, Asoziale, S. 57-61; Julia Hörath, Terrorinstrument der „Volksgemeinschaft“? KZ-Haft für „Asoziale“ und „Berufsverbrecher“ 1933 bis 1937/38, in: ZfG 60 (2012) 6, S. 513-532, hier S. 516-520.

17 Matthias Willing, Das Bewahrungsgesetz (1918-1967). Eine rechtshistorische Studie zur Geschichte der deutschen Fürsorge, Tübingen 2003.

18 Im Folgenden: „Grunderlass vorbeugende Verbrechensbekämpfung“, in: Wolfgang Ayaß, „Gemeinschaftsfremde“. Quellen zur Verfolgung von „Asozialen“ 1933-1945, Koblenz 1998, S. 94-98. Ferner: Patrick Wagner, Volksgemeinschaft ohne Verbrecher. Konzeption und Praxis der Kriminalpolizei in der Zeit der Weimarer Republik und des Nationalsozialismus, Hamburg 1996, S. 258-262; Karl-Leo Terhorst, Polizeiliche planmäßige Überwachung und polizeiliche Vorbeugungshaft im Dritten Reich, Heidelberg 1985, S. 115-145.

19 Hörath, Terrorinstrument; Terhorst, Überwachung, S. 61-114. 
Da die „Schutzhaft“ ab April 1934 bei der KZ-Einweisung aufgrund von Devianz und Delinquenz an Bedeutung verlor, bildeten bis 1937 die jeweiligen länderrechtlichen Varianten der „Vorbeugungshaft“ und der § 20 RFV die wichtigsten Rechtsgrundlagen. Bislang ist die Praxis der Verhängung von KZ-Haft auf Grundlage des §20 RFV lediglich für die Länder Bayern und Baden belegt. ${ }^{20}$ Vermutlich verfügten aber alle Länder des Deutschen Reiches über eigene Bestimmungen zur Anwendung der polizeilichen „Vorbeugungshaft“. ${ }^{21}$ Als Vorlage diente den meisten Landesregierungen der preußische „Vorbeugungshafterlass“ vom 13. November 1933. ${ }^{22}$ Dieser war als polizeirechtliches Pendant zum „Gesetz gegen gefährliche Gewohnheitsverbrecher und über Maßregeln der Sicherung und Besserung“23 vom 24. November 1933 geschaffen worden, das aufgrund dieses engen konzeptionellen Zusammenhangs im Folgenden ebenfalls erörtert werden soll.

\section{Das Fürsorgerecht als Grundlage der KZ-Einweisung von „Asozialen“}

Die ergänzend zur RFV im Jahre 1924 erlassenen „Reichsgrundsätze über Voraussetzung, Art und Maß der öffentlichen Fürsorge“ $(R G)^{24}$ knüpften in ihrem $\S 13$ den Bezug von Wohlfahrtsunterstützung an die Leistungsbereitschaft und geordnete Lebensführung der Bedürftigen. „Bei Arbeitsscheu oder offenbar unwirtschaftlichem Verhalten“ sei die Hilfsbedürftigkeit „strengstens zu prüfen sowie Art und Maß der Fürsorge auf das zur Fristung des Lebens Unerlässliche

20 Das bedeutet allerdings nicht, dass diese Praxis nicht auch für andere Länder des Deutschen Reiches nachgewiesen werden könnte. Bislang fehlen allerdings entsprechende Forschungsvorhaben.

21 Terhorst, Überwachung, S. 101-108.

22 Allerdings wiesen einige länderrechtliche Regelungen der „Vorbeugungshaft“ starke Abweichungen von der preußischen Vorlage auf: Hörath, Terrorinstrument, S. 523-525.

23 Gesetz gegen gefährliche Gewohnheitsverbrecher und über Maßregeln der Sicherung und Besserung vom 24.11.1933, in: RGBl. I, S. 995-999; Christian Müller, Das Gewohnheitsverbrechergesetz vom 24. November 1933, Baden-Baden 1997; Terhorst, Überwachung, S. 61-75.

24 Die RG konkretisierten die Form, in der die Wohlfahrtsunterstützung geleistet, und die Bedingungen, unter denen sie gewährt werden sollten. Sie wurden während der Weimarer Republik mehrfach verändert. Hier liegt die Fassung vom 1.8.1931 zu Grunde, in: RGBl. I, S. 441-445; ferner: Verordnung zur Änderung der Reichsgrundsätze über Voraussetzung, Art und Maß der öffentlichen Fürsorge und zur Ausführung des $\S 85$ des Auswertungsgesetzes vom 1.8.1931, in: ebd., S. 439-441. 
zu beschränken “. ${ }^{25}$ Überdies könne in solchen Fällen die Wohlfahrtsunterstützung ,auf Anstaltspflege“ beschränkt und „offene Pflege““ ${ }^{26}$ also die Gewährung von Geld- und Sachleistungen, verweigert werden. Die konkrete Handhabe dafür lieferte $\S 20$ RFV. Er ermöglichte es den Fürsorgebehörden, in Fällen selbstverschuldeter Arbeitslosigkeit die Unterstützungsempfänger in eine geschlossene „Arbeitsanstalt“ einzuweisen. Auf diese Weise sollte dem „unwirtschaftlichen“ Verhalten ein Riegel vorgeschoben und die Betroffenen an „geregelte Arbeit“ gewöhnt werden. Allerdings überwog der Zwangscharakter deutlich den Erziehungsgedanken. Selbst im innerbehördlichen Sprachgebrauch bürgerte sich der Ausdruck „Arbeitszwang“ für die Maßnahmen nach $\S 20$ RFV ein. ${ }^{27}$ Die Verantwortung für die Auswahl geeigneter „Arbeitsanstalten“ legte die RFV in die Hände der Landesregierungen. Diese nutzten dafür in der Regel die Arbeitshäuser. Im Wortlaut sah $\S 20 \mathrm{RFV}$ vor, dass

\begin{abstract}
wer obwohl arbeitsfähig infolge seines sittlichen Verschuldens der öffentlichen Fürsorge selbst anheimfällt oder einen Unterhaltsberechtigten anheimfallen lässt, [...] von der Verwaltungsbehörde auf Antrag des vorläufig oder endgültig verpflichteten Fürsorgeverbandes oder desjenigen, der dem Fürsorgeverbande die Kosten der Unterstützung zu ersetzen hat, in einer vom Lande als geeignet anerkannten Anstalt oder sonstigen Arbeitseinrichtung zur Arbeit untergebracht werden [kann], wenn er Arbeit beharrlich ablehnt oder sich der Unterhaltspflicht beharrlich entzieht. ${ }^{28}$
\end{abstract}

Als Nachweis des „sittlichen Verschuldens“ und „beharrlichen“ Arbeitsverweigerung werteten die Wohlfahrtsbehörden gemeinhin, wenn ein Unterstützungsempfänger Arbeitsgelegenheiten wiederholt abgelehnt beziehungsweise die Arbeit zwar für kurze Zeit aufgenommen, dann aber die Arbeitsstelle wieder verlassen hatte. Um die Arbeitswilligkeit der Unterstützungsempfänger zu prüfen, nutzten viele kommunale Wohlfahrtsbehörden gezielt die Verhängung von fürsorgerechtlicher Pflichtarbeit nach $\S 19$ RFV. Dieser ermöglichte es „in geeigneten Fällen“, die Unterstützung arbeitsfähiger Personen „durch Anweisung angemessener Arbeit gemeinnütziger Art zu gewähren oder von der Leistung solcher Arbeit abhängig“" zu machen. ${ }^{29}$

In Bayern erkannte das Innenministerium am 16. Oktober 1934 das KZ Dachau als Arbeitsanstalt im Sinne des $\S 20$ RFV an. Vorangegangen waren zum

$25 \S 13$, Art. 1, RG.

26 Ebd., Art. 2.

27 Z. B.: Abschn. VII. „Arbeitspflicht“, § 4, Abs. 1 der Verordnung über Voraussetzung, Art und Maß der öffentlichen Fürsorge vom 12.4.1930, in: Gesetz- und Verordnungs-Blatt für den Freistaat Bayern (1930) 14, S. 117-130, hier S. 130.

28 Baath, Fürsorgepflicht, S. 16.

29 Ebd., S. 15; Ayaß, Asoziale, S. 57-61. 
einen erbitterte Auseinandersetzungen über die ausufernde bayerische „Schutzhaftpraxis“, bei denen vor allem Haftanordnungen in Fällen von „Trunksucht, Misshandlung der Ehefrau, Fangen von Singvögeln, Holzfrevel, [...], unsittliche[m] Lebenswandel, grobe[m] Unfug, Arbeitsscheu“ sowie „asoziale[m] Verhalten" in der Kritik standen. ${ }^{30}$ Zum anderen hatten die bayerischen Behörden seit dem Machtantritt der Nationalsozialisten ihr Vorgehen gegen die Angehörigen sozialer Randgruppen deutlich verschärft, was einen gravierenden Unterbringungsengpass in den Arbeitshäusern und ähnlichen Anstalten nach sich zog. So war das Arbeitshaus Rebdorf schon im September 1933, in Folge der „Bettlerrazzia“, derart überfüllt, dass es im November des Jahres begann, regelmäßig einen Teil seiner Insassen in das KZ Dachau zu überstellen. ${ }^{31}$ Angesichts dieser Entwicklung ermöglichte es die direkte fürsorgerechtliche Regelung der KZ-Einweisung, die Verhängung von „Schutzhaft“ wieder stärker an politische Kriterien zu knüpfen und dennoch gegenüber einer bestimmten Untergruppe der „Asozialen“, den unliebsamen Fürsorgeempfängern, die Repressionen zu verstärken. Gleichzeitig konnten die tradierten Anstalten für geschlossene Fürsorge entlastet werden.

Ein knappes Jahr nach dem Vorstoß der bayerischen Behörden institutionalisierte auch Baden ein ähnliches Vorgehen. Am 20. August 1935 ernannte das dortige Innenministerium das Arbeitshaus Kislau, das in seinen Räumen ein frühes KZ beherbergte, ${ }^{32}$ zum Vollzugsort des fürsorgerechtlichen Arbeitszwangs. Spätestens ab 1936 wurden die Arbeitszwangshäftlinge dann nachweislich im KZ Kislau untergebracht. ${ }^{33}$

Die Verfolgungsgeschichte des Bäckers Rudolf B., der im Mai 1935 auf Antrag des Bezirksfürsorgeverbandes München-Stadt im Alter von 52 Jahren in das KZ Dachau eingeliefert wurde, mag das Einweisungsverfahren nach § $20 \mathrm{RFV}$ illustrieren: Aus der Personenakte des B. bei der Polizeidirektion München geht hervor, dass dieser vermutlich seit 1924 mit kürzeren Unterbrechungen auf Wohlfahrtsunterstützung angewiesen gewesen war. Die Versuche der Wohl-

30 Bayerisches Hauptstaatsarchiv (künftig: BayHStA München), StK 6299/1, Schreiben des Reichsstatthalter in Bayern vom 20.3.1934; Johannes Tuchel, Konzentrationslager. Organisationsgeschichte und Funktion der ,Inspektion der Konzentrationslager' 1934-1938, Boppard a. Rhein 1991, S. 153-158.

31 Ayaß, Asoziale, S. 42; ders., „Gemeinschaftsfremde“, S. XVII; Annette Eberle, Häftlingskategorien und Kennzeichnung, in: Wolfgang Benz/Barbara Distel (Hrsg.), Der Ort des Terrors, Bd. I, München 2005, S. 91-109, hier S. 97 u. 104; Tuchel, Organisationsgeschichte, S. $155-158$.

32 Angela Borgstedt, Kislau, in: Wolfgang Benz/Barbara Distel (Hrsg.), Der Ort des Terrors, Bd. II, München 2005, S. 134-136.

33 Hörath, Terrorinstrument, S. 516-520 u. 529-531. 
fahrtsbehörde, B. zur Arbeit zu zwingen, setzten Ende der 1920er-Jahre ein. Am 14. Juni 1928 stellte das Wohlfahrtsbezirksamt München bei der dortigen Polizeidirektion einen Antrag, Rudolf B. gemäß dem bayerischen „Zigeuner- und

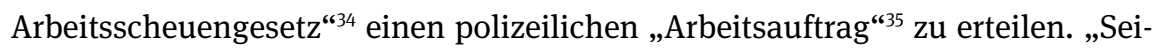
ne Bemühungen um Arbeit“, hieß es in der Begründung,

dürften nicht besonders ernst zu nehmen sein. Zweifellos besteht Arbeitsunwille, weil er in der fraglichen Zeit nicht einmal Gelegenheitsarbeiten aufzuweisen vermag. Bei jeder Antragstellung gebärdet er sich in ungehörigster Weise. Seine Angaben entsprechen nicht immer der Wahrheit. ${ }^{36}$

Wie das polizeiliche Arbeitszwangsverfahren im Jahre 1928 ausging, geht aus der Polizeiakte nicht hervor. Sechseinhalb Jahre später, das NS-Regime hatte sich unterdessen erfolgreich konsolidiert, unternahm das Wohlfahrtsamt einen weiteren Versuch, Rudolf B. zur Arbeit zu zwingen, indem es ihn im November 1934 zum Reichsautobahnbau schickte. Rudolf B. entzog sich diesem „Vermittlungsversuch“ aber, indem er schlichtweg nicht auf der Baustelle erschien. ${ }^{37}$ Daraufhin eröffnete ihm das Wohlfahrtsamt, dass man ihm fortan die Unterstützung nicht mehr in Bargeld auszahlen, sondern nur noch in Form von Anstaltsunterbringung in der Herzogsägmühle ${ }^{38}$ gewähren werde. Angesichts dessen verzichtete Rudolf B. auf den Leistungsbezug und lebte fortan von finanziellen Zuwendungen seiner Mutter.

34 Beim bayerischen „Zigeuner- und Arbeitsscheuengesetz“ handelte es sich um eine länderrechtliche Regelung, die ein sicherheitspolizeiliches Vorgehen gegen sogenannte „Zigeuner“ und „Arbeitsscheue“ ermöglichte. Sie kodifizierte unter anderem den sicherheitspolizeilichen Arbeitszwang, eine dem fürsorgerechtlichen Arbeitszwang nach § 20 RFV ähnliche Maßnahme: Gesetz zur Bekämpfung von Zigeunern, Landfahrern und Arbeitsscheuen vom 16.7.1926, in: Gesetz- und Verordnungs-Blatt für den Freistaat Bayern (1926) 17, S. 359-361.

35 Bayerisches Staatsarchiv München (künftig: BayStA München), C 0402, 1978, Nr.11630, Akte Rudolf B., Wohlfahrtsbezirksamt München VII vom 14.6.1928.

36 Ebd.

37 Bei einer späteren Vernehmung durch die Polizeidirektion München begründete B. sein Nicht-Erscheinen auf der Baustelle damit, dass er die Arbeit beim Reichsautobahnbau aus gesundheitlichen Gründen nicht hätte leisten können. Tatsächlich wurde ihm die Arbeitsunfähigkeit nachträglich amtsärztlich bescheinigt: ebd., Vorführprotokoll der Polizeidirektion München vom 8.5.1935. Dass die Wohlfahrts- und Arbeitsämter bei der Vermittlung die Frage der körperlichen Eignung für die anstrengenden Arbeiten beim Reichsautobahnbau ignorierten, war kein Einzelfall.

38 Bei der Herzogsägmühle handelte es sich um eine Arbeits- und Unterbringungsstätte für Bettler, Landstreicher, Wander- und Saisonarbeiter, die vom Bayerischen Landesverband für Wanderdienst betrieben wurde, der auch eng mit der Politischen Polizei unter Heinrich Himmler und dem KZ Dachau kooperierte: Annette Eberle, Herzogsägmühle in der Zeit des Nationalsozialismus, Peiting 1994. 
Als Vater eines minderjährigen Sohnes, für den er unterhaltspflichtig war, war Rudolf B. langfristig aber nicht vor fürsorgerechtlichen Repressionen sicher, obwohl er selbst nicht im Leistungsbezug stand. Das Kind lebte nämlich auf Wohlfahrtskosten bei einer Pflegemutter. Nachdem der Ortsfürsorgeverband Gräfeling, der Kostenträger, erfolglos versucht hatte, Rudolf B. zu Unterhaltszahlungen heranzuziehen, stellte er am 25. Februar 1935 bei der Polizeidirektion München einen „Antrag auf Einschaffung des Bäckers Rudolf B. in das Arbeitslager Dachau“. ${ }^{39}$ Die Begründung lautete:

\footnotetext{
Der in München [...] wohnhafte [...] Bäcker Rudolf B. ist [...] Vater des Kindes Rudolf S. [...] und soll monatl[ich] 35 RM Unterhaltsbeitrag leisten. Nach Mitteilung der Amtsvormundschaft des Bezirksjugendamts München war es nicht möglich seit Geburt des Kindes auch nur einen Pfennig Unterhalt beizutreiben [...]. [Rudolf B.] ist meistens unbekannten Aufenthalts und wurde wiederholt im Bayer[ischen] Polizeifahndungsblatt ausgeschrieben. B. ist der typische Taugenichts, der sich seiner Unterhaltspflicht mit Absicht entzieht und der daher in ein Arbeitslager gehört. ${ }^{40}$
}

Die Polizeidirektion München reagierte allerdings nur zögerlich auf den Haftantrag. Doch am 24. April 1935, zwei Monate nach dem Gesuch des Ortsfürsorgeverbands Gräfeling, stellte auch das Städtische Wohlfahrtsamt München, bei dem B. kurz zuvor erneut um Unterstützung gebeten hatte, einen Antrag auf „Vollzug des $§ 20$ RFV“. ${ }^{41}$ In der Begründung rekurrierte es auf die schon ein Jahrzehnt währende behördliche Auseinandersetzung mit Rudolf B., die den „Beweis“ erbracht hätte, dass es sich bei B. um einen renitenten Wohlfahrtsunterstützungsempfänger, „Arbeitsverweigerer“ und „säumigen Unterhaltszahler“ im Sinne des § 20 RFV handele. Als Beleg diente in erster Linie eine ausführliche Schilderung der erfolglosen Versuche, Rudolf B. gemäß §19 RFV zu fürsorgerechtlicher Pflichtarbeit heranzuziehen. Als Dauer der Arbeitszwangshaft schlug das Wohlfahrtsamt München-Stadt sechs Monate vor. ${ }^{42}$ Am 8. Mai 1935 eröffnete die Polizeidirektion München Rudolf B. dann den Einweisungsbeschluss. Drei Tage später, am 11. Mai, wurde er in das KZ Dachau überstellt. Entgegen dem Antrag des Wohlfahrtsamts kam B. allerdings schon am 12. August 1935, nach drei Monaten KZ-Haft, wieder frei.

39 BayStA München, C 0402, 1978, Nr. 11630, Akte Rudolf B., Antrag des Ortsfürsorgeverbands Gräfeling vom 25.2.1935.

40 Ebd.

41 Ebd., Antrag des Städtischen Wohlfahrtsamts vom 24.4.1935.

42 Ebd. 


\section{4 „Arbeitsscheue“ in den Begründungen für die Verhängung von „Sicherungsverwahrung“ gegen „Gewohnheitsverbrecher“}

Das „Gewohnheitsverbrechergesetz“ fügte am 24. November 1933 in Form einer Gesetzesnovelle eine Reihe von „Maßregeln der Sicherung und Besserung“ in den $\S 42$ Reichsstrafgesetzbuch (RStGB) ein, die ihrer Konzeption nach auf die sogenannte Zweckstrafe zurückgingen, ${ }^{43}$ für deren Einführung die Strafrechtsreformbewegung zuvor jahrzehntelang gekämpft hatte. Allerdings war die Strafrechtsreform am Ende der Weimarer Republik gescheitert, was nicht zuletzt an der Zersplitterung des Parlaments gelegen hatte, die es als Gesetzgeber nahezu handlungsunfähig machte. Nach dem Ermächtigungsgesetz vom 24. März 1933, das den Exekutivorganen legislative Kompetenzen übertrug, brauchten die Beamten der Ministerialbürokratie die Reformentwürfe nur wieder aus der Schublade zu ziehen, um Teile des Projektes zu realisieren - nun unter Umgehung eines parlamentarischen Gesetzgebungsverfahrens und mit Schwergewicht auf den repressiven Elementen. ${ }^{44}$

Dennoch hob das „Gewohnheitsverbrechergesetz“ den Dualismus von „Sicherung“ und „Besserung“, welcher der Zweckstrafe konzeptionell innewohnte, nicht vollständig auf, sondern kodifizierte auch solche Maßregeln, die hauptsächlich der „Reform“ der Individuen dienen sollten. Die Widerherstellung der Leistungsfähigkeit und -bereitschaft der Betroffenen kann dabei als eines der zentralen Ziele gelten. So war als Maßregel für „notorische“ Bettler und Landstreicher beispielsweise die Arbeitshausunterbringung und für sogenannte „Gewohnheitstrinker“ die Zwangseinweisung in eine Trinkerheilanstalt vorgesehen. Dem lag die lange tradierte Vorstellung zu Grunde, die Individuen mittels Arbeit „bessern“ und an die Anforderungen eines geregelten (Berufs-)Lebens

43 Das Modell der Zweckstrafe hatte der Vordenker der Strafrechtsreformbewegung, Franz von Liszt, maßgeblich entwickelt und propagiert. Im Gegensatz zur Vorstellung der klassischen Strafrechtslehre, in der sich die Strafe allein an der Schwere der Tat zu orientieren hatte, sah die Zweckstrafe eine flexible Anpassung der Strafe an die Persönlichkeit des Täters, die von ihm ausgehende „Gefahr“ und den während der Haft erzielten „Besserungserfolg“ vor. Um die in diesem Sinne geforderten Zusätze im Strafkatalog des RStGB terminologisch von der klassischen Strafe zu unterscheiden, schlug von Liszt vor, von „Maßregeln“ zu sprechen. Innerhalb der Strafrechtslehre setzte sich dann die Formulierung „Maßregeln der Besserung und Sicherung“ durch, welche die Nationalsozialisten 1933 im „Gewohnheitsverbrechergesetz“ in „Maßregeln der Sicherung und Besserung“ verkehrten: Franz von Liszt, Die deterministischen Gegner der Zweckstrafe, in: ZStW 13 (1893), S. 325-370; Müller, Verbrechensbekämpfung, S. 141.

44 Ebd., S. 223-227; Hörath, Terrorinstrument, S. 524f. 
gewöhnen zu können. ${ }^{45}$ Als schärfste und in erster Linie repressiven Zwecken dienende Maßregel sah der neu eingefügte $\S 42 \mathrm{e}$ RStGB vor, dass das Gericht gegen Personen, die als „gefährliche Gewohnheitsverbrecher“ galten, lebenslange „Sicherungsverwahrung“ verhängen konnte. Vollstreckt wurde die „Sicherungsverwahrung“ zunächst in den Justizvollzugsanstalten, ab September 1942 dann in den KZ. ${ }^{46}$

Ähnlich wie in den Begründungen der Fürsorgebehörden zur Verhängung von Arbeitszwang nach $\S 20$ RFV spielten in den Anträgen auf Anordnung der „Sicherungsverwahrung“ neben den formal-juristischen Kriterien der Haftanordnungen ${ }^{47}$ als Legitimationsgrund auch die „Arbeitsscheue“ der Betroffenen eine entscheidende Rolle. So hieß es beispielsweise in einer Antragsbegründung, die der Direktor der Strafanstalt Wittlich/Eifel am 6. Juni 1934 mit Blick auf den dort einsitzenden Sträfling Alex N. formulierte: ${ }^{48}$

\begin{abstract}
N. hat infolge mangelnder Erziehung und erblicher Veranlagung schon mit 17 Jahren die Verbrecherlaufbahn beschritten. Nach dem frühen Tode des Vaters [...] verlor die Mutter gänzlich die Erziehungsgewalt über den Jungen. Er entwickelte sich zu einem arbeitsscheuen, verwahrlosten und sittlich verkommenen Menschen [...]. ${ }^{49}$
\end{abstract}

Dabei bestand in den Augen des Strafanstaltsdirektors bei N. ein direkter Kausalzusammenhang zwischen dessen angeblich angeborenem ,inneren Hang zum Verbrechen“ - nach Auffassung von Kriminologen und zeitgenössischer Rechtsprechung das zentrale Charakteristikum des „gefährlichen Gewohnheitsverbrechers“50 - und dessen „arbeitsscheuem“ Verhalten. Das wiederum machte N. nach Ansicht des Direktors zu einem „minderwertigen Menschen““, ${ }^{51}$ der zu einer „Besserung“ nicht mehr fähig sei:

45 Terhorst, Überwachung, S. $69 f$.

46 Hans-Peter Klausch, „Vernichtung durch Arbeit“, in: KZ-Gedenkstätte Neuengamme (Hrsg.), Ausgegrenzt. „Asoziale“ und „Kriminelle“ im nationalsozialistischen Lagersystem, Bremen 2009, S. 60-71.

47 Für eine Darstellung der Anordnungsvoraussetzungen: Terhorst, Überwachung, S. 65-71.

48 Da das „Gewohnheitsverbrechergesetz“ unter bestimmten Bedingungen sogar die rückwirkende Anordnung der „Sicherungsverwahrung“ ermöglichte, durchkämmten die Strafanstaltsdirektionen kurz nach dessen Inkrafttreten systematisch die Insassen der Justizvollzugsanstalten nach geeigneten Kandidaten: Sylvia de Pasquale, Zwischen Resozialisierung und „Ausmerze“. Strafvollzug in Brandenburg an der Havel (1920-1945), Berlin 2013, S. 134 u. 390-396.

49 Landesarchiv Nordrhein-Westfalen/Abteilung Rheinland (künftig: LAV NRW R), BR 2034 VH I, Nr. 15, Akte Alex N., Antrag des Strafanstaltsdirektors in Wittlich/Eifel [vom 6.7.1934].

50 Entscheidungen des Reichsgerichts in Strafsachen, Berlin 1934, S. 155; ebd., Berlin 1939, S. 295.

51 LAV NRW R, BR 2034 VH I, Nr. 15, Akte Alex N., Antrag des Strafanstaltsdirektors in Wittlich/Eifel [vom 6.7.1934]. 
N. ist der Typ des Verbrecherführers, den keine noch so harte Strafe von seiner Verbrecherlaufbahn abbringen wird. Er ist ein intelligenter, selbstsicherer, aber verbissener Mensch, bei dem keine Zeichen innerer Umstellung oder Wille hierzu festzustellen sind. Er hat eine ausgesprochene Scheu gegen geregelte, ehrliche Arbeit und zieht den mühelosen Lebenserwerb durch Verletzung fremden Eigentums vor. Er hält sich selbst für den geborenen Verbrecher und glaubt nicht an die Möglichkeit einer Besserung. Er gehört zu den moralisch und sozial minderwertigen Menschen, in denen das natürliche Ehrgefühl gestorben ist und die sich nicht mehr zu einem gesetzmäßigen Leben aufraffen können. ${ }^{52}$

Im Fall von Alex N. lehnte der zuständige Staatsanwalt den Antrag auf „Sicherungsverwahrung“ allerdings ab. Doch verhängte die Kriminalpolizei Köln am 29. Dezember 1934 „Vorbeugungshaft“ gegen N. und veranlasste seine Überstellung in das KZ Lichtenburg.

\section{5 „Arbeitsscheue“ in den Begründungen für die Verhängung von „Vorbeugungshaft" gegen „Berufsverbrecher"}

Die „Vorbeugungshaft“ war in Preußen nahezu zeitgleich mit dem „Gewohnheitsverbrechergesetz“ durch den Erlass zur „Anwendung der vorbeugenden Polizeihaft gegen Berufsverbrecher"53 vom 13. November 1933 geschaffen worden. Zum zentralen Definitions- und Erkennungsmerkmal des „Berufsverbrechers“ erhob der Erlass das aus „Gewinnsucht“ begangene Verbrechen, das im Denken von Kriminologen und Strafrechtsreformern traditionell eng mit der „Arbeitsscheue“ der Zielpersonen zusammenhing. Schon Robert Heindl, der mit seinem 1926 publizierten Buch Der Berufsverbrecher den Weimarer Diskurs über die „Vorbeugenden Verbrechensbekämpfung“ entscheidend prägte, ${ }^{54}$ hatte hervorgehoben, dass für den „Berufsverbrecher“ nicht Arbeit, sondern das Begehen von Straftaten Mittel zum Broterwerb sei, so wie für „,andere Menschen schustern, schneidern [und] Bücher schreiben“ ${ }^{.55}$ Der preußische Erlass sah dann die „Vorbeugungshaft“ für Personen vor, die „der Kriminalpolizei als Berufsverbre-

52 Ebd.; Thomas Roth, „Verbrechensbekämpfung“ und soziale Ausgrenzung im nationalsozialistischen Köln. Kriminalpolizei, Strafjustiz und abweichendes Verhalten zwischen Machtübernahme und Kriegsende, Köln 2010, S. 203.

53 Geheimes Staatsarchiv Preußischer Kulturbesitz (künftig: GStAPK), I. HA. Rep. 84a, Justizministerium, Nr. 8203, Geheimerlass über die „Anwendung der vorbeugenden Polizeihaft gegen Berufsverbrecher" vom 13.11.1933.

54 Wagner, Volksgemeinschaft, S. 19-25.

55 Heindl, Berufsverbrecher, S. 164. 
cher bekannt“ waren und „die ausschließlich oder zum größten Teil vom Erlöse aus Straftaten“ lebten. ${ }^{56}$ Darüber hinaus galt als „äußere Voraussetzung“ für die Haftanordnung, „dass der Betroffene dreimal wegen eines aus Gewinnsucht begangenen vorsätzlichen Verbrechens oder Vergehens zu Zuchthaus oder Gefängnis von mindestens sechs Monaten verurteilt worden“ war. ${ }^{57}$

Der enge innere Zusammenhang, den der kriminologische und strafrechtliche Diskurs zwischen dem Streben nach einem „mühelosen Lebenserwerb durch Verletzung fremden Eigentums“ einerseits und der „ausgesprochenen Scheu gegen geregelte, ehrliche Arbeit“ andererseits herstellte, ${ }^{58}$ hilft zu erklären, warum so viele Haftanträge als Nachweis für die „Gewinnsucht“ der Zielperson auf deren „arbeitsscheues“ Verhalten rekurrierten. So hieß es beispielsweise in einer Haftbegründung, welche die Kriminalpolizei Duisburg am 24. März 1934 für Christian H. verfasste, dieser sei ein ,arbeitsscheuer Mensch und Einbrecher“, der „seinen Unterhalt [...] zum größten Teil aus dem Erlös strafbarer Handlungen“ bestreite. ${ }^{59}$

Nicht selten spielte die Frage, ob eine als „Berufsverbrecher“ verdächtigte Person in einem geregelten Arbeitsverhältnis stand beziehungsweise grundsätzlich bemüht war, ein solches aufzunehmen, schon im Vorfeld der Haftanordnung eine wichtige Rolle. So begründete die Münchener Polizei ihre Erwägung, Paul B., einen 35-jährigen Korbmacher, wenige Monate nach dessen Entlassung aus dem Zuchthaus Straubing in „Vorbeugungshaft“ zu nehmen, wie folgt:

Seine Arbeitsscheue und verbrecherische Gesinnung lassen berechtigte Zweifel aufkommen, dass er nunmehr den Willen aufbringt, sich von seiner früheren Lebensweise abzuwenden und einer geordneten und nachprüfbaren Arbeit nachzugehen. Es ist vielmehr zu vermuten, dass sein arbeitsscheues Verhalten in Hohenbercha, [dorthin war B. kurz zuvor verzogen, J. H.] sehr bald Anstoß erregen und zu neuerlichen Straftaten führen wird. ${ }^{60}$

In den Augen der Polizei galt Paul B. als „brutaler Charakter“, der seine Straftaten mit „größter Rohheit“ ausführte. ${ }^{61}$ Tatsächlich war er insgesamt sechzehn Mal vorbestraft, darunter wegen Raubs in Tateinheit mit gefährlicher Körperverletzung, aber auch wegen Sachbeschädigung, Hausfriedensbruchs, grobem

56 GStAPK, I. HA. Rep. 84a, Justizministerium, Nr. 8203, Geheimerlass über die „Anwendung der vorbeugenden Polizeihaft gegen Berufsverbrecher“ vom 13.11.1933.

57 Ebd.

58 LAV NRW R, BR 2034 VH I, Nr. 15, Akte Alex N., Antrag des Strafanstaltsdirektors in Wittlich/Eifel [vom 6.7.1934]; vgl.: Roth, „Verbrechensbekämpfung“, S. 203.

59 LAV NRW R, BR 1111, Nr. 96, Akte Christian H.

60 BayStA München, C 0402, 1978, Nr.11704, Akte Paul B., Schreiben der Polizeidirektion München vom 6.5.1936.

61 Ebd., Kriminalpolizei München, „Krimineller Lebenslauf“ vom 26.4.1936. 
Unfugs und verbotenem Rauchens. Allerdings konnte die Polizei, als sie den Haftantrag stellte, Paul B. keine konkrete Straftat nachweisen. Das war zur Verhängung von „Vorbeugungshaft“ aber auch gar nicht notwendig, handelte es sich doch um eine straftatunabhängige Präventivmaßnahme.

Um dennoch begründen zu können, dass die Zielperson „vom Erlös aus Straftaten“ lebte, also unter die Anordnungsvoraussetzungen der „Vorbeugungshaft“ fiel, musste häufig das ,arbeitsscheue Verhalten“ herhalten - insbesondere dann, wenn es mit einem finanziell aufwendigen Lebensstil einherging, dessen Quelle der Polizei suspekt war. So war eines der wenigen Verdachtsmomente, die die Kriminalpolizei Duisburg gegen Erich F., den sie der Zuhälterei bezichtigte, vorzubringen hatte, dass dieser immer „sehr nobel gekleidet“ sei und „stets als vornehmer Mann in Erscheinung“ trete. ${ }^{62}$ Über Paul B. wusste die Kriminalpolizei München zu berichten, dieser hätte „ein bequemes Leben“ geführt, ,trotzdem er seit seiner Entlassung aus dem Zuchthaus keiner geregelten Arbeit“ nachginge. Daher bestünde „der dringende Verdacht, dass B. aus dem Erlös strafbarer Handlungen seinen Unterhalt bestreitet". ${ }^{63}$ Kurz nach der Verhaftung hieß es dann, B. wäre „,bestimmt nicht in vorbeugende Polizeihaft genommen worden“, wenn er einen „geregelten Arbeitsnachweis gehabt“ hätte. ${ }^{64}$ Am 4. Juni 1936 überstellte die Polizeidirektion München Paul B. in das KZ Dachau. Dort verlor sich drei Jahre später seine Spur.

\section{Die „Arbeitsleistung“ als Grund der KZ-Entlassung}

Eine ähnliche Bedeutung wie bei der Haftanordnung kam dem Aspekt der Leistungsbereitschaft beziehungsweise „Arbeitsscheue“ auch für die Entlassungspraxis zu. Erwägungen, ob die „Arbeitserziehung“ im KZ den angestrebten „Besserungseffekt“ erzielt habe und ob die Person im Anschluss an die KZ-Haft in ein festes Arbeitsverhältnis vermittelt werden könne, ${ }^{65}$ spielten bei den regelmäßigen Haftprüfungen eine ausschlaggebende Rolle. So schrieb die Kom-

62 LAV NRW R, BR 1111, Nr. 155, Akte Erich F.

63 BayStA München, C 0402, 1978, Nr.11704, Akte Paul B., Schreiben der Polizeidirektion München von März 1936.

64 Ebd., Schreiben der Gendarmeriestation Karlshud vom 26.5.1936.

65 Gemäß den Durchführungsrichtlinien zum „Grunderlass vorbeugende Verbrechensbekämpfung“ von April 1938 galt neben der Zustimmung des Reichskriminalpolizeiamtes (RKPA) das Vorhandensein einer Arbeitsstelle als unabdingbare Voraussetzung für die Entlassung aus der „Vorbeugungshaft“, in: Ayaß, Gemeinschaftsfremde, S. 71. 
mandantur des KZ Buchenwald am 3. Mai 1938 über den „Vorbeugungshäftling“ Heinrich A.:

Die Führung und Arbeitsleistung des A. sind hier nicht zufriedenstellend. Wird er nicht dauernd unter Aufsicht gehalten, führt er die ihm übertragenen Arbeiten nur nachlässig und mangelhaft aus. [...] Es besteht nicht der Eindruck, dass A. aufgrund der bisherigen Vorbeugungshaft gebessert worden ist. ${ }^{66}$

Anderen „Vorbeugungshäftlingen“ bescheinigten die Lagerleitungen hingegen tatsächlich einen „Besserungserfolg“. So schrieb die Kommandantur des KZ Flossenbürg am 27. Februar 1940 anlässlich eines Haftprüfungstermins über den „Berufsverbrecher“ Alfred G.:

Die Führung ist genügend, seine Arbeitsleistungen entsprechen den hier gestellten Anforderungen. G. verrichtet willig und fleißig die ihm übertragene Arbeit. Es besteht hier der Eindruck, dass G. in der Volksgemeinschaft noch ein brauchbarer Arbeiter wird, wenn er sofort nach seiner Entlassung in den Arbeitsprozess eingegliedert wird. ${ }^{67}$

Nachdem die Lagerleitung ein Jahr später diesen Eindruck in einem weiteren Führungsbericht bekräftigte, hob der Reichsführer-SS und Chef der deutschen Polizei Heinrich Himmler die „Vorbeugungshaft“ auf. Alfred G. wurde am 7. April 1941 aus dem KZ Flossenbürg entlassen.

\section{Die Bedeutung von Einsatzfähigkeit, Arbeitsbereitschaft und Leistungsverweigerung bei den Massenrazzien 1937/38}

Im Vorangegangenen hoffe ich deutlich gemacht $\mathrm{zu}$ haben wie grundlegend die Diskurse um Leistungsbereitschaft und „Arbeitsscheue“ für die KZ-Einwiesungen von „Asozialen“ und Mehrfachstraftäter waren. Und auch 1937/38, als der unter Heinrich Himmler zentralisierte Polizeiapparat ${ }^{68}$ das Vorgehen gegen diese beiden Verfolgtengruppen enorm intensivierte, spielten Fragen der Ar-

66 Archiv des International Tracing Service Bad Arolsen, KZ Flossenbürg - individuelle Unterlagen, Akte Heinrich A., Führungsbericht der Kommandantur KZ Buchenwald vom 3.5.1938.

67 Ebd., Akte Alfred G., Führungsbericht der Kommandantur KZ Flossenbürg vom 27.2.1940. Ich danke Sven Langhammer für den Hinweis auf die beiden ITS-Akten.

68 Am 17. Juni übertrug Hitler die Befehlsgewalt über die gesamte Polizei an Heinrich Himmler. Dieser konnte sich fortan Reichsführer-SS und Chef der Deutschen Polizei (RFSSuChDtPol) nennen. 
beitswilligkeit beziehungsweise Leistungsverweigerung in den einschlägigen Befehlen eine entscheidende Rolle. So definierten die Richtlinien des RKPA zum „Grunderlass vorbeugende Verbrechensbekämpfung“ als „Asoziale“ unter anderem „Personen, [...] die sich der Pflicht zur Arbeit entziehen und die Sorge für ihren Unterhalt der Allgemeinheit überlassen" ${ }^{69}{ }^{69}$ Der erste Himmler-Befehl zur April-Welle der „Aktion Arbeitsscheu Reich“ im Jahre 1938 visierte dementsprechend als Zielgruppe explizit Männer an, die obgleich arbeitsfähig, „,nachweisbar in zwei Fällen die ihnen angebotenen Arbeitsplätze ohne berechtigten Grund abgelehnt oder die Arbeit zwar aufgenommen, aber nach kurzer Zeit [...] aufgegeben“70 hatten. Und sowohl von der März-Aktion gegen „Berufsverbrecher“ im Jahre 1937 als auch von der zweiten Verhaftungswelle der „Aktion Arbeitsscheu Reich“ im Juni 1938 waren explizit Personen ausgenommen, die in einem festen Arbeitsverhältnis standen. ${ }^{71}$

Wie dargelegt, hatten diese Haftkriterien Vorläufer, die weit in die Zeit vor 1933 zurückreichten: Schon die $\S \S 19$ und 20 RFV hatten die aus „sittlichem Verschulden“ resultierende Hilfsbedürftigkeit arbeitsfähiger Personen zur Anordnungsvoraussetzung der fürsorgerechtlichen Pflichtarbeit und des Arbeitszwangs gemacht. Dabei galt das mehrmalige Ablehnen eines Beschäftigungsangebots traditionell als Nachweis für die „beharrliche“ Arbeitsverweigerung des Unterstützungsempfängers. Und umgekehrt schloss schon das bayerische „Zigeuner- und Arbeitsscheuengesetz“ von 1926 bei Bestehen eines geregelten Arbeitsverhältnisses die Anordnung des sicherheitspolizeilichen Arbeitszwangs aus.

In der historiographischen Debatte über die Ausweitung der Verfolgungspraxis 1937/38 sind jene Haftkriterien, die die Einsatzfähigkeit, Arbeitsbereitschaft beziehungsweise Leistungsverweigerung der Zielgruppe adressierten, meines Erachtens nach bislang zu einseitig als Beleg für die ökonomischen Motive der Massenrazzien im Zuge der Mobilisierung für den Vierjahresplan interpretiert worden. Derartige Deutungen stützen sich im Wesentlichen auf einen einzelnen Hinweis auf den Vierjahresplan, der sich in einem Befehl Reinhard Heydrichs von 1. Juni 1938 findet, mit dem dieser die zweite Welle der „Aktion Arbeitsscheu Reich“ anordnete, ${ }^{72}$ sowie auf ein Zitat des Leiters der Vierjah-

69 Richtlinien des RKPA über die Durchführung der vorbeugenden Verbrechensbekämpfung vom 4.4.1938, zit. n.: Vorbeugende Verbrechensbekämpfung. Erlaßsammlung, [Berlin] 1941, S. 71.

70 Schreiben des RFSSuChDtPol vom 26.1.1938, zit. n.: ebd., S. 47.

71 Schreiben des RFSSuChDtPol vom 23.2.1937, zit. n.: ebd., S. 28. Schreiben des RKPA vom 1.6.1938, zit. n.: ebd., S. 82.

72 Schreiben des RKPA vom 1.6.1938, zit. n.: ebd. 
resplanbehörde, Ulrich Greifelt, der ein Jahr nach der Verhaftungsaktion retrospektiv deren Ziele und sich selbst als Initiator des polizeilichen Vorgehens beschrieb. ${ }^{73}$ Erklärungsansätze, die diese zwei Belegstellen zum Ausgangspunkt einer Gesamtinterpretation der Massenverhaftungen 1937/38 nehmen, übersehen jedoch die hier dargelegten Diskurstraditionen und überkommenen Exklusionspraktiken und laufen Gefahr, zu Gunsten einer rein auf die ökonomischen Entwicklung fokussierten Perspektive jene Radikalisierungsdynamiken zu vernachlässigen, die der rassistischen und kriminalpräventiven Logik selbst innewohnten. $^{74}$

\section{Fazit}

Die Kategorie Arbeit ist eine Grundkonstante bürgerlich-kapitalistischer Gesellschaften. Am Beispiel der Verfolgung und KZ-Einweisung von sogenannten „Asozialen“ und Mehrfachstraftätern kann gezeigt werden, wie das NS-Regime sowohl auf der diskursiv-ideologischen Ebene als auch in der Praxis an das bürgerliche Arbeitsethos und dessen Leistungsnormen anknüpfte, indem es beispielsweise bestehende Ausgrenzungs- und Disziplinartechniken wie die Arbeitshäuser, den fürsorgerechtlichen Arbeitszwang oder auch die von den Strafrechtsreformern vor 1933 propagierten Konzepte aufnahm und ins Terroristische wendete.

Die Analyse der Fallbeispiele hat deutlich gemacht, dass die Kategorie Arbeit tief in die Konstituierung der Gesellschaft und ihrer Subjekte eingelassen war: Weder die Normierung des gesellschaftlich erwünschten Verhaltens, verkörpert im Bürger oder „Volksgenossen“, noch die Entwürfe von deren Gegenbildern, den „Asozialen“, „Verbrechern“ oder „Gemeinschaftsfremden“, ${ }^{75}$ waren ohne den Rekurs auf die Kategorie Arbeit denkbar. Alle Personen, die das Regime als „gemeinschaftsfremd“ einstufte, einte, dass man ihnen „Arbeitsscheue“ unterstellte und zwar unabhängig davon, ob sich ihre konkreten Normverletzungen in der Renitenz gegenüber Behörden, einem abweichenden Sexualverhalten, Gesetzesverstößen oder übermäßigen Alkoholkonsum manifestierte.

73 Für eine Darstellung der daran geknüpften Interpretation: Ayaß, Asoziale, S. 163.

74 Für eine Überblick und eine Kritik an den bisherigen Interpretationsansätzen: Wagner, Volksgemeinschaft, S. 254-298.

75 Zum Begriff „Gemeinschaftsfremde“: Wolfgang Ayaß, „Demnach ist zum Beispiel asozial...“. Zur Sprache sozialer Ausgrenzung im Nationalsozialismus, in: BGN (2012) 28, S. 69-89. 
Gleichzeitig waren die Repressionen gegen „Asoziale“, „Berufs“- und „Gewohnheitsverbrecher" aber auch ein probates Mittel, um bestimmte wirtschaftliche Aktivitäten im Bereich der Schattenökonomie zu kontrollieren oder ganz zu unterbinden: So wollte man das Sex-Gewerbe polizeilich reglementieren. Eine der Strategien war die Inhaftierung von Prostituierten und Zuhältern als „Asoziale“, „Berufs“- oder „Gewohnheitsverbrecher“. Des Weiteren versuchten die Behörden, die Scharen von Arbeitsmigranten, die in Gestalt von Wander-, Saison- und Gelegenheitsarbeitern seit der Weltwirtschaftskrise die deutschen Landstraßen bevölkerten, in „geordnete Bahnen“ zu lenken. Ebenfalls bekämpft werden sollten die Praktiken vieler „Wanderer“ und Unterschichtsangehöriger, die ihren Lebensunterhalt vollständig oder teilweise durch das Erbitten von Almosen bestritten. Die „Bettlerrazzia“ im September 1933 bildete diesbezüglich nur den medienwirksamen Auftakt einer Verfolgungspraxis, die sich in den folgenden Jahren verstetigte und radikalisierte, bis sie in den Massenrazzien der „Aktion Arbeitsscheu Reich“ 1938 einen weiteren Höhepunkt fand.

All das zeigt, dass der Kategorie Arbeit bei der Formierung der „Volksgemeinschaft" eine ausschlaggebende Bedeutung zukam: Sie war Kriterium des gesellschaftlichen Ein- beziehungsweise Ausschlusses - ein Kriterium das über die Verteilung von (Über-)Lebenschancen entschied. 